\title{
Design and Production Considerations on a Button Type Beam Position Monitor for Use of TAC-TARLA Facility
}

\author{
M. Tural Gundogan ${ }^{a, *}$, C. KAyA ${ }^{b}$ And O. YAVAS $^{a}$ \\ ${ }^{a}$ Department of Physics Engineering, Ankara University, 06100 Tandogan, Ankara, Turkey \\ ${ }^{b}$ Institute of Accelerator Technologies, Ankara University, 06830 Golbasi, Ankara, Turkey \\ (Received January 23, 2017; revised version May 10, 2017; in final form October 16, 2017)
}

Turkish Accelerator and Radiation Laboratory in Ankara (TARLA) facility is a superconducting linac-based infrared free-electron laser light source in the frame of Turkish Accelerator Center (TAC). Construction of the facility premises was completed in 2009, with a continued progress of hardware and infrastructure installation until today. In this respect, a button type beam position monitor (BPM) is designed, produced and tested for use of TARLA facility. Antenna simulations and mechanical design studies based on TARLA electron beam parameters are carried out by the software computer simulation technology. In this paper, its design and production highlights are discussed in detail, and the original results are summarized.

DOI: 10.12693/APhysPolA.132.1333

PACS/topics: 41.60.Cr, 29.27.-a, 29.27.Fh, 07.10.-h, 07.57.-c

\section{Introduction}

The Turkish Accelerator Center (TAC) project is an inter-university collaboration with twelve Turkish universities, under the coordination of Ankara University [1]. The TAC project aims to establish accelerator and light source facilities in Turkey for scientific research and technological developments in basic and applied sciences, funded by Turkish Ministry of Development.

The TARLA oscillator free electron laser (FEL) facility is currently under construction in Golbaşı campus of Ankara University [2]. It is aimed to produce FEL in the range of 3-250 $\mathrm{\mu m}$ wavelengths with a repetition rate of $13 \mathrm{MHz}$. The TARLA consists of a DC thermionic triode. The DC Thermionic gun produces electron pulses with a charge up to $77 \mathrm{pC}$ and electron energy of $250 \mathrm{keV}$. The electron pulse length is about of 500 ps RMS at the gun output. The bunches are compressed along the injector section by ballistic compression down to 10 ps using two buncher cavities (BC) and long drift path. Further they will be captured by the first cavity of the first linac and the bunch lengths will be reduced to $2 \mathrm{ps}$. According to operation mode, the bunches will be either compressed or decompressed between 0.4 and 6 ps by using bunch compressor between the two linac modules. The first buncher (sub-harmonic) operates at $260 \mathrm{MHz}$, the second one operates at the fundamental frequency of LINAC which is $1.3 \mathrm{GHz}[3]$.

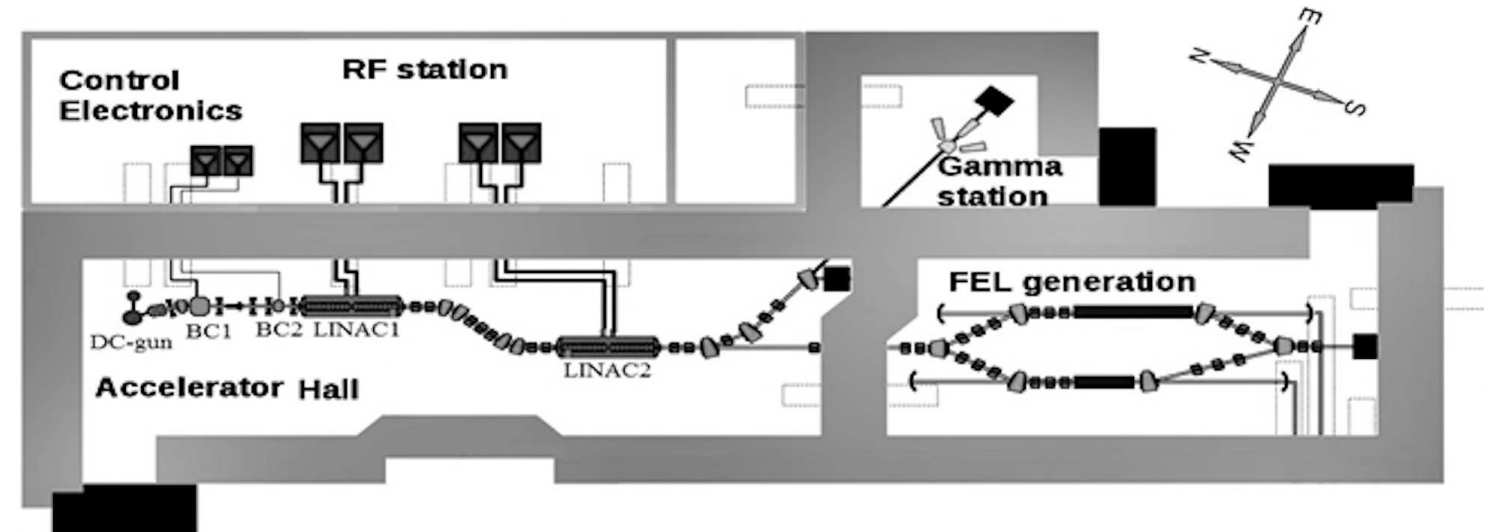

Fig. 1. Installation plan of the TARLA.

TARLA is based on two superconducting electron linear accelerating modules. Each module consists of two TESLA type nine-cell cavities. Each TESLA cavity is designed to operate with an accelerating field gradient of $10 \mathrm{MV} / \mathrm{m}$ so that the maximum design of electron beam energy at the exit of the second module is $40 \mathrm{MeV}$.
TARLA delivers an electron beam with an average current of up to $1 \mathrm{~mA}$. Figure 1 [4] shows a schematic installation plan of the TARLA facility.

TARLA FEL hall will consist of two separated optical cavities, each housing one undulator, U25 and U90, respectively. Considering tunability, high power and short 
pulse properties of FEL, it is planned to built-up various user instruments for scientific research and technological applications in material science, nonlinear optics, semiconductors, biotechnology, medicine, and photochemical processes. In addition, a Gamma station is also planned for nuclear spectroscopy studies with high energy gamma beams between 5 and $35 \mathrm{MeV}$. Main parameters of the TARLA facility are given in Table I [5].

\section{TABLE I}

Main parameters of the TARLA Facility.

\begin{tabular}{l|c}
\hline \hline Parameter & Value \\
\hline energy [MeV] & $15-40$ \\
\hline max. bunch charge [pC] & 77 \\
average beam current [mA] & 1.0 \\
bunch repetition rate [MHz] & 13 \\
bunch length (before linacs) [ps] & $500-10$ \\
bunch length (after linacs) [ps] & $0.4-6$ \\
norm. RMS trans. emittance [mm mrad] & $<16$ \\
norm. RMS long. emittance [keV ps] & $<100$ \\
macro pulse duration [ps] & $50-\mathrm{cw}$ \\
macro pulse rept. rate [Hz] & $1-\mathrm{cw}$
\end{tabular}

In particle accelerators, many diagnostics tools are used to measure beam parameters such as beam energy, beam position, beam current, beam charge etc. The beam passing through the beam pipe should be kept under control. If the beam deviates from its position, it can cause radiation damages on beam pipe wall and other diagnostic devices. Beam position monitor (BPM) is one of the most important beam diagnostic device to detect position of the electron bunches inside the beam pipe [6].

$\mathrm{BPM}$ is a device to measure the electronic charge induced by electric field of the electron beam on an insulated metal plate. The four plates should be placed facing each other around the beamline to measure the exact position of the beam. Different signals measured from facing plates give information about the position of the beam. There are various BPM types such as: button, stripline and cavity type, which are mostly used in accelerator facilities. Each type of BPM has different advantages and challenges [7].

It is planned that $40 \mathrm{BPMs}$ (i.e. button and stripline type) will be used in TARLA beamline because the beam repetition frequency and average current of TARLA are high enough to get measurable signals on electrodes of the monitors. For ps bunches, button type BPMs are more effective than other types. Thus, button type BPM design, production and test studies are performed in this study.

\section{Basic principles of a button type beam position monitor}

A button type BPM consists of four small and circular electrodes placed facing one another under vacuum. The voltage values generated on isolated electrodes are recorded in vertical and horizontal positions. The difference of voltages gives information of the beam position indirectly.

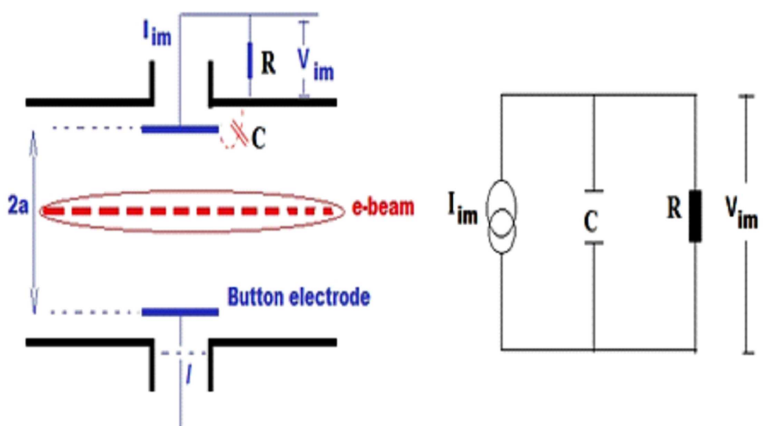

Fig. 2. Geometry and an equivalent circuit for a button BPM [9].

A geometry and an equivalent diagram for a button BPM is given in Fig. 2. A surface wall current is induced when the beam passes through the beam pipe [8]. This wall current is monitored by the button electrode inserted in the beam pipe. $Q_{i m}$ is the image charge and $I_{i m}$ is the image current driven by the image charge. The bunch current is

$$
\begin{aligned}
& \frac{\mathrm{d} Q_{\text {beam }}(t)}{\mathrm{d} t}=\frac{\mathrm{i} \omega l I_{\text {beam }}(w)}{\beta c} \frac{\mathrm{d} I_{\text {beam }}}{\mathrm{d} t}=\frac{l}{\beta c} \frac{\mathrm{d} Q_{\text {bunch }}(t)}{\mathrm{d} t} \\
& =\frac{l}{\beta c} \frac{\mathrm{d} I_{\text {bunch }}}{\mathrm{d} t}=\frac{l}{\beta c} \mathrm{i} \omega I_{\text {bunch }}(w),
\end{aligned}
$$

where the electron beam velocity $v=\beta c$. The bunch current $I_{\text {bunch }}$ is a function of angular frequency $\omega$ and given as $I_{\text {bunch }}=I_{0} \mathrm{e}^{\mathrm{i} \omega t}[9]$. The voltage $V_{i m}$ is measured across a resistor $R$. The button capacitance $C$ and the load resistance $R$ define the circuit impedance given by Eq. (2):

$$
Z_{t}=\frac{R}{1+\mathrm{i} \omega R C} Z=\frac{R}{1+\mathrm{i} \omega R C} .
$$

The longitudinal transfer impedance on BPM voltage is described by this impedance $Z_{t}$. BPM transfer impedance

$$
Z_{t}=\frac{A}{2 \pi \beta c C a} \frac{\omega / \omega_{\text {cut }}}{\sqrt{1+\omega^{2} / \omega_{\text {cut }}^{2}}},
$$

where the symbols have their usual meanings: frequency $(\omega)$, velocity of particles $(\beta)$ and geometrical factors (i.e. the length of button plate $l$, area of button plate $A$, and distance of two opposite buttons $2 a$ ). $\omega_{\text {cut }}$ is cut-off frequency, $c$ is the speed of light [9].

In Fig. 3, a basic diagram of a button type BPM is shown. The $x$-axis position is recorded by the buttons 2 and 4 , and the $y$-axis position is recorded by buttons 1 and 3. Beam position is obtained by comparing the voltages of either horizontal or vertical directions, and then registered to the electronic control system.

The deviations of the electron beam on the $x$ and $y$ axes are given by Eq. (4) and Eq. (5), where $V_{x}$ and $V_{y}$ are voltage signals obtained from facing buttons. $S_{x}$ and $S_{y}$ are the position sensitivities on $x$-axis and $y$-axis 


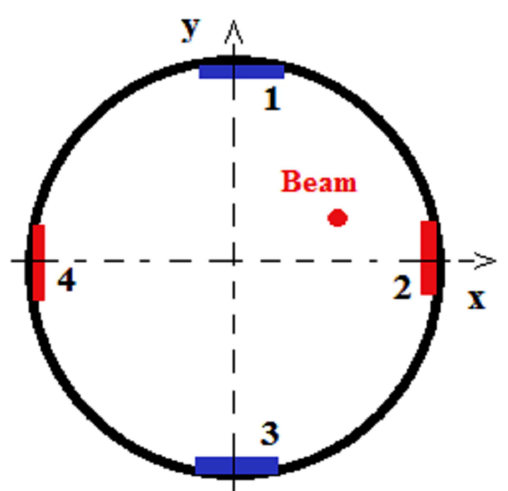

Fig. 3. Basic diagram of a button type BPM.

respectively. $\delta_{x}$ and $\delta_{y}$ are offset corrections in units of $\mathrm{mm}$; namely they are the higher order terms

$$
\begin{aligned}
& x=\frac{1}{S_{x}} \frac{V_{2}-V_{4}}{\Sigma V_{x}}+\delta_{x} \equiv \frac{1}{S_{x}} \frac{\Delta V_{x}}{\Sigma V_{x}}+\delta_{x}, \\
& y=\frac{1}{S_{y}} \frac{V_{1}-V_{3}}{\Sigma V_{y}}+\delta_{y} \equiv \frac{1}{S_{y}} \frac{\Delta V_{y}}{\Sigma V_{y}}+\delta_{y} .
\end{aligned}
$$

\section{Simulation results by the software CST studio}

In order to determine the position of the beam, 4 identical buttons are located in the beamline as shown in Fig. 4. Sizes of the buttons are chosen by considering electron beam parameters of the TARLA facility such as beam size, bunch current and bunch length. DN40 of pipe size has been foreseen at TARLA beamline. Although we could have chosen $38 \mathrm{~mm}$ aperture for BPMs, $35 \mathrm{~mm}$ has been chosen because of the limitations of antenna ports which were standard products of any vendor.

CST Studio 2014 code [10] on hexahedral mesh was used in numerical simulations of the button type BPM. The optimal sizes of the button antennas were determined using this code. First, a circular button antenna was drawn and simulated by CST Studio. For simulation; a Gaussian bunch with a velocity of $\beta=0.9999$ and a FWHM (for a Gaussian distribution FWHM = $2 \sqrt{2 \ln 2} \sigma$ ) value of $3 \mathrm{~mm}$ (having a bunch charge of $1 \mathrm{pC}$ ), is applied along the $+x$ axis of a circular beam pipe. BPM input voltage in time domain is shown in Fig. 5. The manufactured BPM will be used after the BC1 (Fig. 1) and here the bunch length varies between 10 and 40 ps. Therefore, 30 ps pulse was considered with CST simulation.

Electron bunch charges obtained from thermionic gun are adjustable between 1 and $77 \mathrm{pC}$. It is a challenge to measure minimum bunch charge for many diagnostic applications. Therefore, simulation was done for $1 \mathrm{pC}$ electron bunch to design a BPM that can measure all bunch charge values between 1 and $77 \mathrm{pC}$.

Button radii were varied from $2 \mathrm{~mm}$ to $4.5 \mathrm{~mm}$ at $0.5 \mathrm{~mm}$ intervals. BPM output voltage was registered with the voltage monitor defined at the end of the button antennas. In Fig. 6, output voltages at different button radii are shown.

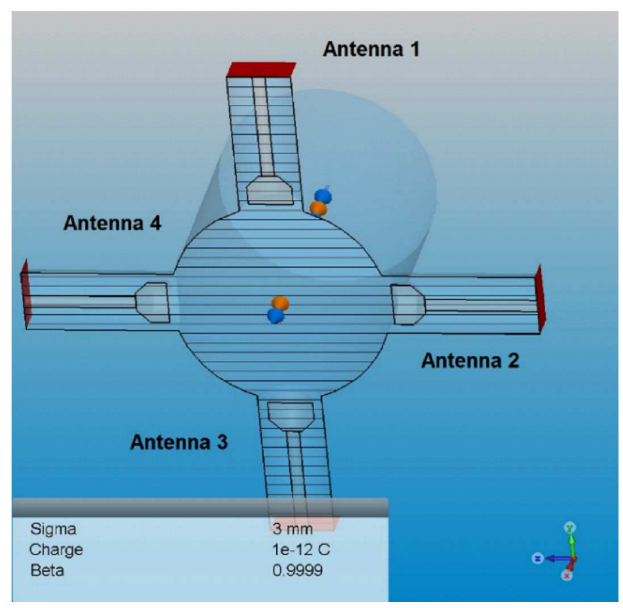

Fig. 4. Technical drawing of a button type BPM by CST Studio.

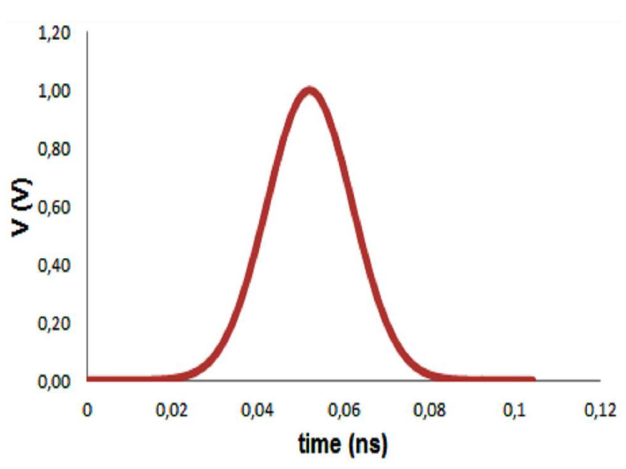

Fig. 5. BPM input voltage in time domain.

On the other hand, simulation was done at different bunch charges to see the variation of the output signal versus the bunch charge. It is planned to measure a minimum bunch charge of $1 \mathrm{pC}$ for TARLA, resulting in a BPM measurement capability between 1 and 100 pC. Figure 7 shows output signals at different bunch currents. As the applied current increases, the amplitudes of the signals obtained from the button antennas increase linearly.

Quality of the signal is crucial for signal processing. The signal power $P_{s}$ at different button radius is calculated according to Eq. (6) below. The coefficient $A$ is determined by the shape of bunch. Because of the Gaussian bunch $A$ was taken as 1 and $R$ is the coaxial impedance. The distance of the opposite buttons is $2 a$ and the button radius is $r$. $f$ is the bunch frequency of $1.3 \mathrm{GHz}$ and $I$ is the average current of $1 \mathrm{~mA}$

$$
P_{s}=\frac{2 \pi^{2} a^{4}}{r^{2} \beta^{2} c^{2}} R A^{2} f^{2} I^{2} .
$$

The noise power is given by Eq. (7). $B$ is measurement bandwidth of $1 \mathrm{MHz}, k_{\mathrm{B}}$ is the Boltzmann coefficient and $T$ is the temperature of $300 \mathrm{~K}$. It is calculated as $-114 \mathrm{~dB}$.

$$
P_{n}=k_{\mathrm{B}} T R B \text {. }
$$

The signal-to-noise ratio (SNR) was calculated using Eq. (8). In Table II, the calculated SNR parameters are shown at different radii in $\mathrm{dB}$ for the bunch 
charge of $1 \mathrm{pC}$

$$
S N R=\frac{P_{s}}{P_{n}} .
$$

TABLE II

SNR parameters at different radii.

\begin{tabular}{c|c|c}
\hline \hline$r[\mathrm{~mm}]$ & $P_{s}[\mathrm{dBm}]$ & $\mathrm{SNR}[\mathrm{dB}]$ \\
\hline 2 & -60.05 & 53.95 \\
2.5 & -56.18 & 57.82 \\
3 & -53.01 & 60.99 \\
3.5 & -50.32 & 63.68 \\
4 & -48.01 & 65.99 \\
4.5 & -45.97 & 68.03
\end{tabular}

For different bunch charges and optimum button radius which is $3.5 \mathrm{~mm}$, SNR parameters were calculated using Eqs. (6)-(8). The calculated SNR parameters are given in Table III.

TABLE III

SNR parameters at different bunch charges.

\begin{tabular}{c|c|c}
\hline \hline$q[\mathrm{pC}]$ & $P_{s}[\mathrm{dBm}]$ & SNR $[\mathrm{dB}]$ \\
\hline 1 & -90.42 & 23.58 \\
10 & -70.42 & 43.58 \\
25 & -62.91 & 51.09 \\
50 & -56.88 & 57.12 \\
75 & -53.27 & 60.73 \\
100 & -50.42 & 63.58
\end{tabular}

The SNR values vary depending on the signal power measured from the antennas. In Table II it is seen that the SNR values increase as the antenna radius increases. However, in Fig. 6 it is seen that the amplitude of measured signal at the radius of $3.5 \mathrm{~mm}$ is larger than the amplitude of measured signals at the radii of $4 \mathrm{~mm}$ and $4.5 \mathrm{~mm}$. According to the SNR parameters it is observed that $3.5 \mathrm{~mm}$ button radius is optimum for both signal quality and signal amplitude as well. Similarly, Table III shows that the SNR values increase as the bunch charge increases. Figure 7 shows that the lowest signal amplitude was obtained from $1 \mathrm{pC}$ bunch charge. The SNR value at $1 \mathrm{pC}$ is a measurable value.

The button output signal depends on the frequency range as compared to the cut-off frequency. The value of the button capacitance has to be known to calculate the cut-off frequency. The electric field energy $\varepsilon$, obtained by using CST EM Studio is used to calculate button capacitance

$$
\varepsilon=\frac{1}{2} V^{2}
$$

$1 \mathrm{~V}$ is applied on each button plate (see Fig. 5), and $\varepsilon$ is obtained by CST EM Studio. In Fig. 8, the distribution of applied voltages is shown with different colors. The electric field energy is calculated to be $2.16 \times 10^{-12} \mathrm{~J}$. According to Eq. (9), $4.33 \mathrm{pF}$ button capacitance is calculated by using obtained electric field and applied po-

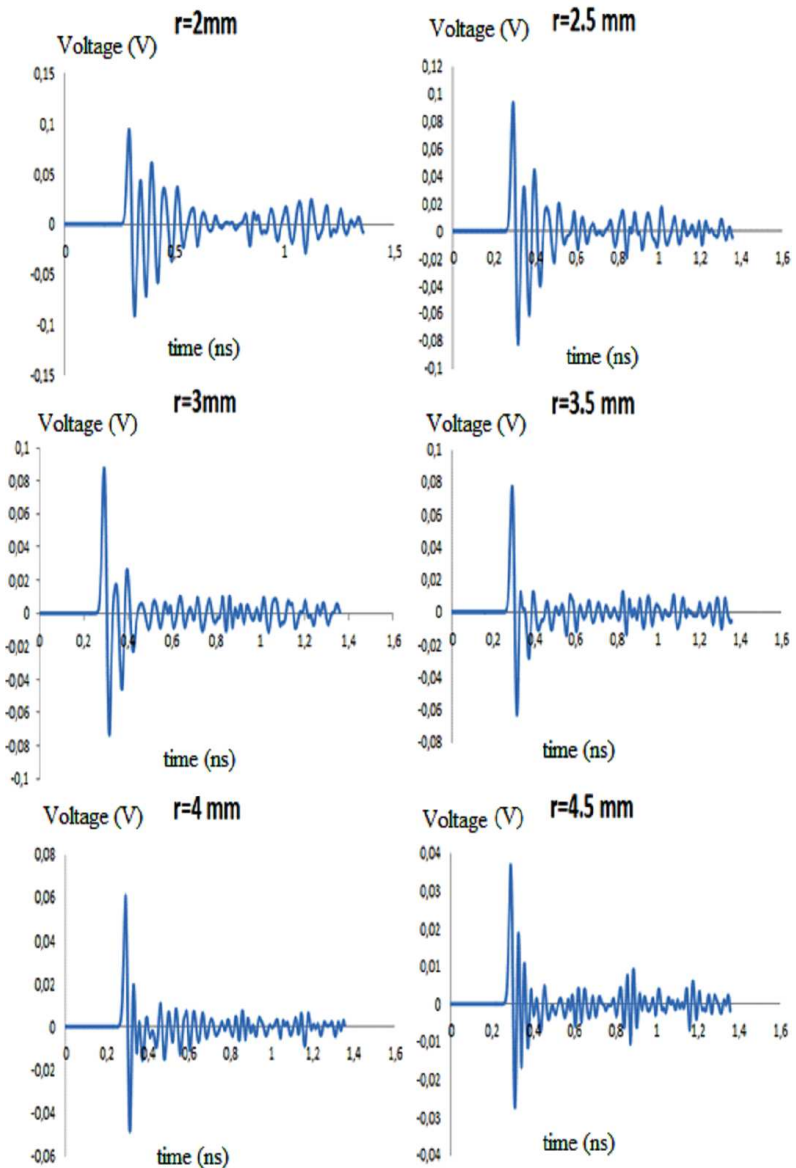

Fig. 6. BPM output voltages in time domain at different radii.

tential. The cut-off frequency depends on capacitance and cable impedance $R$ by Eq. (10):

$$
f_{\text {cut }}=\frac{1}{2 \pi R C} \text {. }
$$

$50 \Omega$ is characteristic impedance of the BPM cable. The high impedance termination $50 \Omega$ resistor leads to smooth signal transmission. It prevents signal reflections on the transmission line. For TARLA, the cut-off frequency is calculated as $735 \mathrm{MHz}$. Bunch frequency is $13 \mathrm{MHz}$, which is significantly lower than the cut-off frequency. In this case, transfer impedance will have a capacitive effect. A higher button voltage is generated with a smaller capacitance according to Eq. (3). In Fig. 9, button transfer impedance distribution versus frequency is given ( $R=50 \Omega$ for low impedance).

In order to calculate the resolution of the BPM, the applied signal position was changed by $1 \mathrm{~mm}$ steps from $-13 \mathrm{~mm}$ to $+13 \mathrm{~mm}$ along the $x$-axis, and then the output signals versus displacement were registered. $\Delta V / \Sigma V$ was calculated by Eq. (4). In Fig. 10, $\Delta V_{x} / \Sigma V_{x}$ vs. displacement of applied signal is shown. It is called resolution curve of BPM.

The slope of the linear region in the plot as seen in Fig. 10 gives the position sensitivity $S_{x}$ for $x$-axis. Ac- 


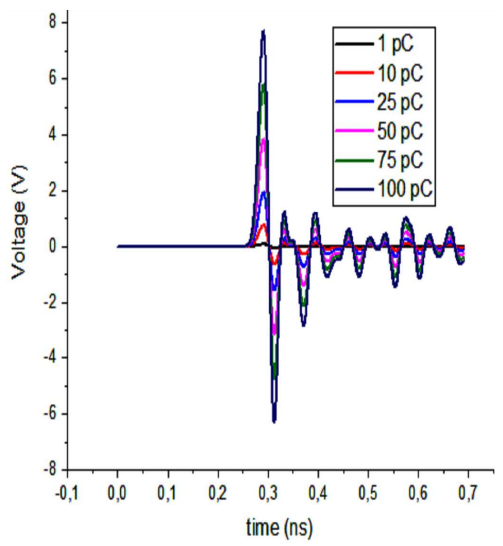

Fig. 7. BPM output voltages at different bunch currents.

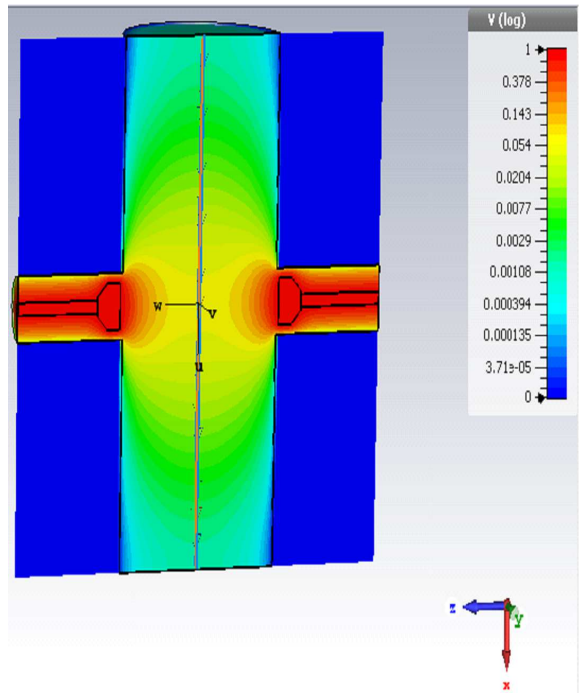

Fig. 8. Distribution of the applied $1 \mathrm{~V}$ voltage on the buttons.

cording to CST Studio simulations, the position sensitivity is $110 \mathrm{~dB} / \mathrm{mm}$. The position sensitivity is proportional to the beam displacement and the signal strength.

\section{Mechanical production of the button type BPM}

The optimization studies of BPM were simulated by CST Studio Suite. It was manufactured by a company (DORA Machinery Company) which resides in Turkey's high-tech manufacturing industrial zone, OSTİM. The $3.5 \mathrm{~mm}$ button BPM includes a main BPM body, four antennas and flanges, CF 40 and CF 16. Main body, antennas and flanges are assembled under ultra-high vacuum. A photo of manufactured main body, antenna and whole BPM is shown in Fig. 11a respectively and the solid view of designed main body, antenna and whole BPM is shown in Fig. 11b. Each button plate has a radius of $3.5 \pm 0.05 \mathrm{~mm}$ and the distance between opposed button plates is $35 \pm 0.5 \mathrm{~mm}$.

The main body is made of AISI 316 stainless steel material. On the other hand, antenna material is made of

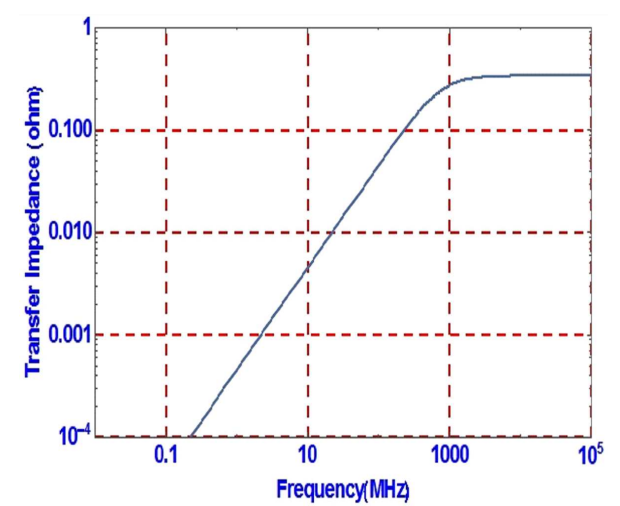

Fig. 9. Button transfer impedance vs. frequency.

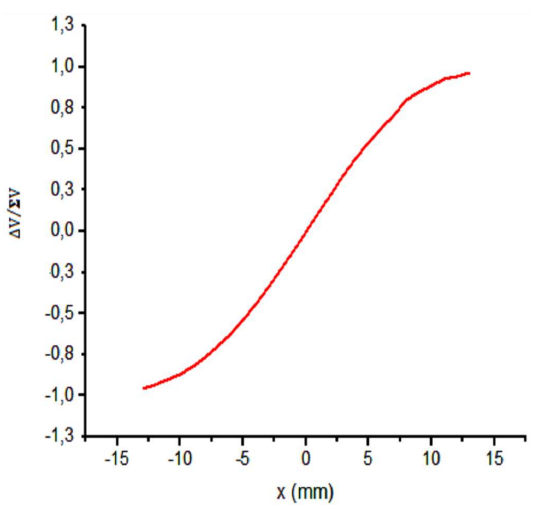

Fig. 10. The resolution curve of simulated BPM.

copper with golden coating in order to prevent the oxidation of copper. The helium leak rate of all manufactured goods is less than $10^{-10} \mathrm{mbar} \mathrm{l} / \mathrm{s}$. Tungsten inert gas (TIG) welding was applied and no leakage on the welding seam was detected. The antenna was mounted on the main body using a copper gasket to decrease the leakage rate. The front view of designed BPM is given in Fig. 12a and the side view is given in Fig. $12 \mathrm{~b}$.

\section{BPM bench test results}
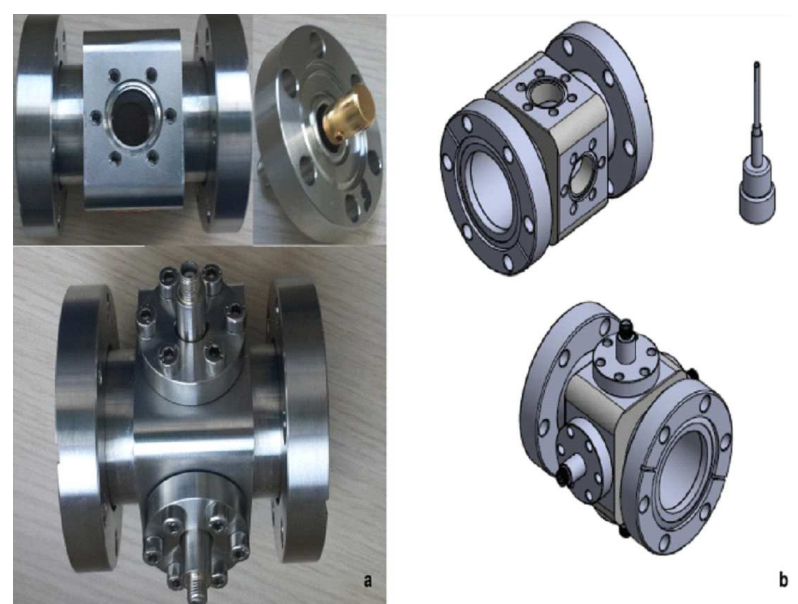

Fig. 11. (a) Photos of manufactured pieces of BPM, (b) solid view of the designed pieces BPM. 

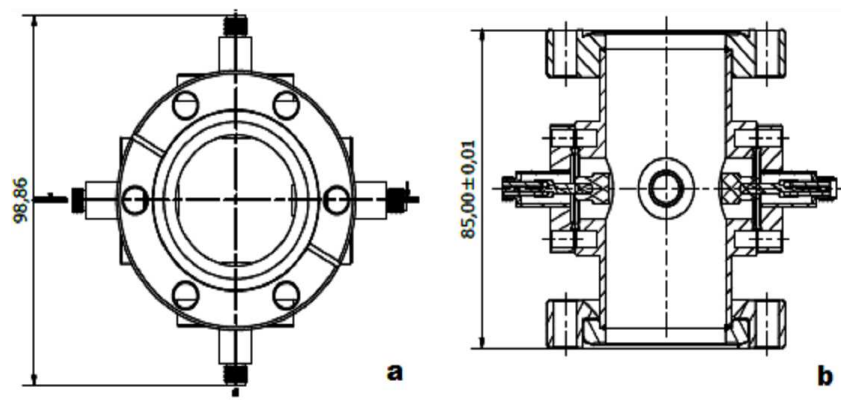

Fig. 12. The technical drawings of BPM: (a) the front view, (b) the side view.

The bench tests of the BPM were made by a test setup at TARLA facility. It consists of a Tektronics AFG3101 signal generator, Avtech pulse source, and an Agilent $500 \mathrm{MHz}$ scope. A thin wire was installed inside the BPM. Bunch repetition rate for TARLA is $13 \mathrm{MHz}$. This produces Gaussian pulses at the interval of $77 \mathrm{~ns}$. To obtain a signal of $1 \mathrm{~mA}$ on the test bench the $510 \Omega$ resistor was used. The signal applied to the wire is seen in Fig. 13. The oscilloscope screen image was taken using a $40 \mathrm{~dB}$ attenuator. The amplitude of the applied signal is $80 \mathrm{~V}$.

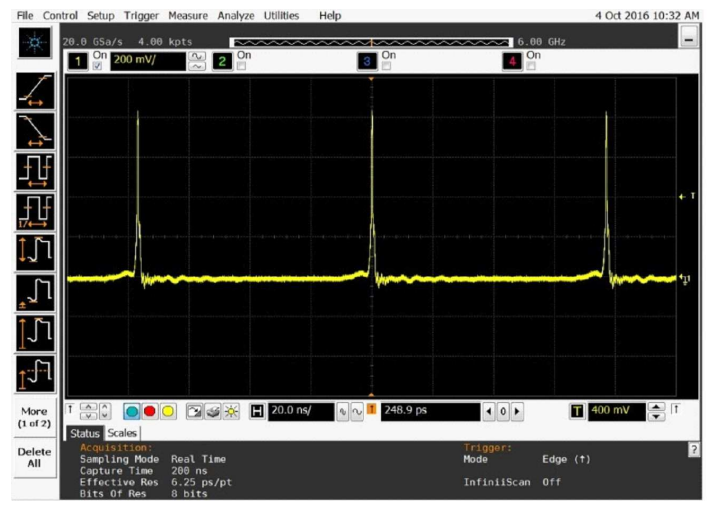

Fig. 13. The image screen of the applied signal to the wire.

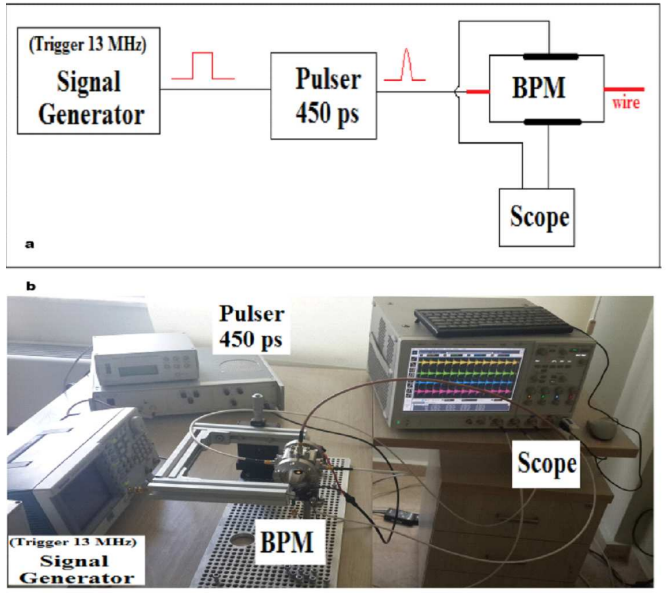

Fig. 14. (a) Block diagram of the test setup, (b) a photo of TARLA BPM test setup.

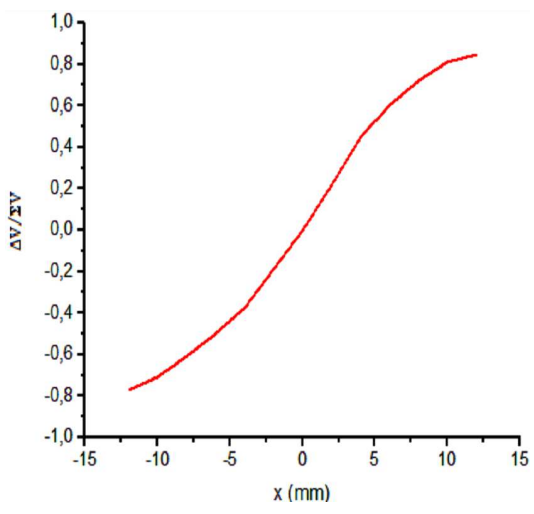

Fig. 15. The resolution curve of manufactured BPM.

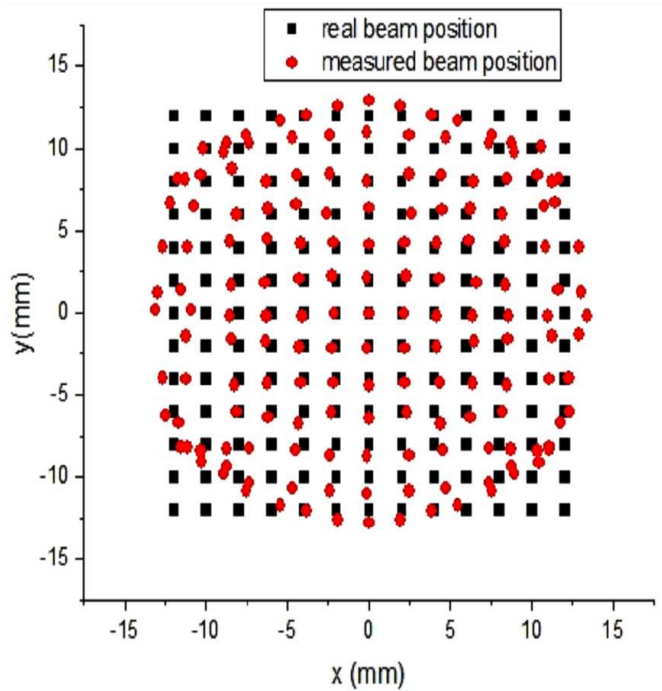

Fig. 16. 2D mapping of BPM response.

The block diagram of the test setup is shown in Fig. 14a, and a photo of BPM test setup is shown in Fig. 14b, respectively.

The position of the wire was changed by $2 \mathrm{~mm}$ steps from $-12 \mathrm{~mm}$ to $+12 \mathrm{~mm}$ in $x$-axis, and then the output signals vs. displacement were registered by scope. In Fig. 15, $\Delta V / \Sigma V$ vs. displacement of tested signal is shown. The position sensitivity was obtained to be $103 \mathrm{mV} / \mathrm{mm}$ referring to the test results. It is seen that the position sensitivity value obtained as a result of the simulation is in agreement with the results obtained in the bench tests. This indicates that the simulated BPM is compatible with the manufactured BPM.

Conversion of the signals registered by four buttons to the actual beam position is done by Eq. (4) and Eq. (5). Figure 16 shows 2-dimensional mapping of BPM response. A position map was created to determine the measured beam position inside the beam pipe. This map provides information about the accuracy of the beam position measurement as it passes through the BPM. Each red point in the map corresponds to the measured position of the beam, and each black point corresponds to the real beam position. 
Thin wire displacement error range is $\pm 10 \mu \mathrm{m}$ of the linear translation stage which has 3 axes. The linear range of the stage varies in $\pm 7 \mathrm{~mm}$, the range cover approximately $40 \%$ of the measurement area of BPM, as it is seen in Fig. 16. Nonlinearities have been seen outside that range according to Fig. 16. These are caused by the higher order terms given in Eq. (4) and Eq. (5). Therefore, the measurements is made between $\pm 7 \mathrm{~mm}$ where the distributions are linear (see Fig. 16). However, in order to measure the beam displacement above $40 \%$ of beam pipe it is possible to use numerical methods (i.e. interpolation, curve fitting, etc.).

\section{Conclusions}

A button type BPM is designed, manufactured and tested, referring to TARLA electron beam parameters. Design of TARLA button type BPM antenna performance is numerically simulated by CST Particle Studio. The radius of the button antennas is the most important restriction. The antenna sizes, which give the maximum amplitude and high quality output signal, were so chosen to obtain accordance with the simulation results. Position sensitivity of simulated BPM was obtained to be $110 \mathrm{mV} / \mathrm{mm}$ by simulations. For $3.5 \mathrm{~mm}$-radius button BPM, the button capacitance $\mathrm{C}$ was calculated as $4.33 \mathrm{pF}$. Such a small capacitance value allows measuring higher voltages. Bench test of the BPM was made in TARLA facility. The position sensitivity of the button BPM is obtained to be $103 \mathrm{mV} / \mathrm{mm}$ by test measurements. Simulation and test results for position sensitivity differ from each other. This difference is due to the mechanical production and the test setup itself. One important parameter is the accuracy and it is affected by the BPM mechanical tolerances. BPM pickups will have an electrical error relative to the mechanical center of the vacuum chamber. The button pickups were designed in a single piece to minimize the manufacture errors. Mechanical tolerances of the BPM pickups are $\pm 0.05 \mathrm{~mm}$. The misalignment between the electrical and the mechanical center will be found experimentally with the wire test pulse.

A position map was created for BPM. Button BPM was designed to determine the beam position in a region with a $35 \mathrm{~mm}$ radius. In $\pm 7 \mathrm{~mm}$ of the BPM, the beam position can be determined linearly. It is sufficient for measuring short bunches. Because the bunch charge is adjustable between 1 and $77 \mathrm{pC}$, it is necessary to correct all nonlinearities for all bunch charge values as numerically or electronically. This is not a practical method, so it would be more effective to follow the beam position obtained linearly online.

The produced button BPM will be used after the first module to measure the beam position. It is also planned to be used in the end of the injector for experimental purposes. Due to the fact that TARLA beamline radius is $38 \mathrm{~mm}$, different types of BPMs with large beamline radiuses are required. In other words, stripline type BPM and cavity type BPM studies are also ongoing for the TARLA facility.
Both simulation results and test set up measurements are fairly well consistent. It is expected that success in BPM production will motivate Turkish industry to develop and produce other advanced components of particle accelerators such as RF cavities, vacuum components, cooling, diagnostic and control tools etc. Once the TARLA facility is commissioned successfully, it will undoubtedly trigger construction of other light source proposals of the Turkish Accelerator Center (TAC) project, like TURKAY and TURKSEL.

\section{Acknowledgments}

The authors would like to thank Turkish Ministry of Development for funding TARLA facility, under grant No. DPT2006K-120470. Authors also thank to Dr. Ayhan Aydin for technical support about the test setup, Dr. Bora Ketenoğlu and Dr. Ozlem Karsli for fruitful discussions.

\section{References}

[1] Turkish Accelerator Center (TAC) Collaboration.

[2] Turkish Accelerator and Radiation Laboratory in Ankara (TARLA).

[3] A. Aksoy, U. Lehnert, Nucl. Instrum. Methods Phys. Res. A 762, 54 (2014).

[4] A. Aksoy, O. Karsl,, The Technical Design Report of Turkish Accelerator and Radiation Laboratory in Ankara, (TARLA), Ankara University, Institute of Accelerator Technologies, Ankara 2015.

[5] A. Aydın, E. Kasap, Turk. J. Phys. 41, (2017).

[6] M. Minty, CERN Accelerator School: Course on Synchrotron Radiation and Free-Electron Lasers, Brunnen, Switzerland 2003, p. 239.

[7] R.E. Shafer, Beam Position Monitoring, AIP Conf. Proc., 1989, p. 212.

[8] A. Lunin, N. Eddy, T. Khabiboulline, V. Lebedev, V. Yakovlev, in: Proc. IBIC2013, Oxford, (UK), 2013, p. 392.

[9] P. Forck, P. Kowina, D. Liakin, Proc. CERN Accelerator School on Beam Diagnostics, Dourdan, France 2008, p. 187.

[10] CST Studio Code. 